\title{
Progressive locomotor recalibration during blind walking
}

\author{
John W. Philbeck, Adam J. Woods, And Joeanna Arthur \\ George Washington University, Washington, D.C. \\ AND \\ JENNIFER TODD \\ University of Maryland, Baltimore, Maryland
}

\begin{abstract}
Blind walking has become a common measure of perceived target location. This article addresses the possibility that blind walking might vary systematically within an experimental session as participants accrue exposure to nonvisual locomotion. Such variations could complicate the interpretation of blind walking as a measure of perceived location. We measured walked distance, velocity, and pace length in indoor and outdoor environments $(1.5-16.0 \mathrm{~m}$ target distances). Walked distance increased over 37 trials by approximately $9.33 \%$ of the target distance; velocity (and to a lesser extent, pace length) also increased, primarily in the first few trials. In addition, participants exhibited more unintentional forward drift in a blindfolded marching-in-place task after exposure to nonvisual walking. The results suggest that participants not only gain confidence as blind-walking exposure increases, but also adapt to nonvisual walking in a way that biases responses toward progressively longer walked distances.
\end{abstract}

Over the last quarter century, walking has been increasingly used as a means of measuring perceived object locations (e.g., Allen, Kirasic, Rashotte, \& Haun, 2004; CreemRegehr, Willemsen, Gooch, \& Thompson, 2005; Loomis, Da Silva, Philbeck, \& Fukusima, 1996; Philbeck, Loomis, \& Beall, 1997; Rieser, Ashmead, Talor, \& Youngquist, 1990; Thomson, 1980; Wu, Ooi, \& He, 2004, among a host of others). As this method is typically used, participants view a target under well-lit viewing conditions, then cover their eyes and attempt to walk without assistance to the remembered target location. This method is known as visually directed walking, open-loop walking, and blind (or blindfolded) walking. Under well-lit viewing conditions, accuracy is generally high for target distances up to at least $22 \mathrm{~m}$, although there is some undershooting for larger distances (Andre \& Rogers, 2006). This good accuracy has been taken as evidence that observers accurately perceive the target location as seen from their initial viewpoint (Loomis et al., 1996; Philbeck \& Loomis, 1997). This interpretation relies upon the assumption that the walking is well calibrated, so that the distance observers walk accurately reflects their perceived target distance.

Recent work has shown that exposure to nonvisual locomotion can itself affect the calibration of nonvisual locomotion (Durgin et al., 2005; Rieser, Pick, Ashmead, \& Garing, 1995). The impact of this on blind walking as a measure of perceived distance is currently unknown. This is an important issue for at least two reasons. First, in many blind-walking studies, participants are provided with several minutes of exposure to nonvisual walking prior to testing, often on the assumption that this will increase the participants' feelings of confidence and safety while walking blindfolded (Bigel \& Ellard, 2005; CreemRegehr et al., 2005; Elliott, 1987; Mohler et al., 2007; Philbeck \& Loomis, 1997; Rieser et al., 1995; Steenhuis \& Goodale, 1988). Increased confidence may improve response precision and reduce any tendency to stop walking sooner than intended, due to hesitancy. Another effect of the exposure to nonvisual walking, however, may be to alter participants' locomotor calibration prior to testing, meaning that the walked distance may not accurately reflect the perceived target location during the experiment proper. Second, in blind-walking experiments, participants accumulate exposure to nonvisual locomotion while generating their responses. If exposure to nonvisual walking alters the calibration of the walking responses themselves (e.g., by creating a general tendency to walk farther and farther for each target as the experiment unfolds), it is possible that the impressive accuracy typically observed in blind-walking studies is, to some degree, an artifact of recalibration that takes place during the experiment.

Our definition of calibration in the context of blind walking is quite broad: It is the degree of correspondence between the physical target location and the distance walked when one attempts to walk to the target location. In a wellcalibrated blind-walking response, a participant would arrive accurately at the physical target location while walking without vision. Under this definition, a wide variety of factors, both peripheral (e.g., motor) and central (e.g., cognitive), could potentially affect the calibration of loco-

J.W. Philbeck, philbeck@gwu.edu 
motion. In this article, we will examine several of these possible factors. First, participants may simply change their response strategy as they gain experience with nonvisual locomotion. This might happen, for example, because they begin to concentrate on different aspects of the task, or because their overall level of concentration changes. They might also have a subjective sense of walking inaccurately in earlier trials (although they receive no objective error feedback) and may consciously respond differently in subsequent trials to correct for this. These factors clearly would be considered cognitive influences on calibration. Second, increasing exposure to nonvisual walking may help participants gain confidence with their ability to walk safely while blindfolded. A common assumption (never tested directly, to our knowledge) is that observers may tend to stop short of their intended destination or behave more erratically if they lack confidence in their safety (Sun, Campos, Young, Chan, \& Ellard, 2004). Indeed, this sort of conservative strategy, whether conscious or unconscious, might be advantageous for survival. At any rate, many walking studies provide participants with at least some exposure to nonvisual walking prior to testing (e.g., $3 \mathrm{~min}$ ), on the assumption that this will maximize the observers' feelings of confidence and safety. This would be another cognitive influence on calibration.

A third possible source of changes in walking calibration comes from perceptuomotor adaptation. Several investigators (Anstis, 1995; Durgin \& Pelah, 1999) have found that when individuals run without vision for several minutes, either on solid ground or on a treadmill, and then attempt to run in place without vision, they involuntarily drift forward. A similar aftereffect occurs when participants attempt to walk (or "march") in place while blindfolded after walking on a treadmill, while viewing a stationary scene in a helmet-mounted display (Proffitt, Stefanucci, Banton, \& Epstein, 2003). Other researchers have found a tendency to involuntarily rotate when participants attempt to march in place without vision after walking on a rotating turntable (Melvill Jones, Fletcher, Weber, \& Block, 2005; Pick, Rieser, Wagner, \& Garing, 1999). In addition to these drift aftereffects, Durgin, Pelah, and Amiruddin (1998) found substantial increases in a blind-walking task after observers ran without vision on a treadmill, relative to their pretreadmill performance (see also Rieser et al., 1995). Durgin and colleagues have argued that all these results reflect the operation of adaptation processes that seek to minimize the discrepancy between motor predictions of self-motion and perceived self-motion. They point out that self-motion perception is based on input from multiple sensory systems and that different sensory cues may be brought into conflict under certain situations. During nonvisual walking, the motor system registers that the individual is moving (e.g., via proprioceptive feedback and efference copy), while the absence of vision may be treated as a cue signaling zero self-motion. In this view, the absence of vision decreases the amount of self-motion that is actually perceived, and this creates a discrepancy between perceived self-motion (a composite signal encompassing multiple sensory systems) and the amount of self-motion signaled specifically by the motor system.
The effective gain of the kinesthetic self-motion signals is then attenuated in order to reduce this mismatch (Barlow, 1990). Durgin et al. (2005) refer to this as "adaptation of self-motion perception," to emphasize the importance of the multimodal character of perceived self-motion and to deemphasize the importance of mismatches between kinesthetic and visual signals in particular. Opinions vary as to whether this type of recalibration should be considered peripheral or central (a point that we will discuss in more detail in Experiment 2, below), but, nevertheless, adaptation of this kind can explain not only the drift aftereffect, but also systematic changes in blind-walking tasks after participants walk on a treadmill (Anstis, 1995; Durgin et al., 2005; Rieser et al., 1995).

Although several studies have demonstrated systematic changes in blind walking after exposure to nonvisual walking on a treadmill, currently it is unclear what impact exposure to nonvisual walking on solid ground might have on subsequent blind-walking responses. Elliott (1987) reported that exposure to nonvisual walking just prior to beginning a blind-walking experiment tended to reduce undershooting in the experiment proper, but the source of this change has not been systematically investigated. As we have argued, modifications of the calibration of walking might occur due to changes in conscious strategies, confidence, or adaptation. These factors are not mutually exclusive and might, thus, operate simultaneously to varying degrees. We conducted the following experiments to assess these possibilities. In Experiment 1, we confirmed that exposure to nonvisual walking on solid ground prior to engaging in a blind-walking task is, indeed, associated with longer walking responses. In Experiment 2, we explored the source of this effect, focusing primarily on the role of adaptation processes.

\section{EXPERIMENT 1}

Using a blind-walking task, Proffitt et al. (2003) found that participants walked significantly farther after exposure to walking on a treadmill than they did prior to the treadmill walking. There is also evidence (Elliott, 1987) that exposure to nonvisual walking on solid ground is associated with an increase in blind-walking responses. Before proceeding, we wanted to confirm the apparent influence of nonvisual walking on subsequent blind-walking performance. Participants in the familiarization phase of this experiment walked for 3 min either with or without vision and then completed a test phase in which they viewed targets and attempted to walk to them without vision. If exposure to nonvisual walking indeed recalibrates walking, due either to increased confidence in the task or to adaptation, walked distances should be longer after nonvisual walking in the familiarization phase. To minimize the possibility that there might be recalibration of walking during the experimental trials themselves, the number of walking trials was kept low (12 trials).

Because the participants always walked for $3 \mathrm{~min}$ (either with or without vision) during the familiarization phase, physiological factors during the familiarization phase were held constant, leaving only the presence or absence of vision 
during locomotion to vary. The visual information available during the test phase did not differ depending on whether participants walked with or without vision during familiarization, so it was unlikely that visual perception of the target distance would differ systematically after visual versus nonvisual familiarization. Nevertheless, to check this, we had the participants on some trials verbally estimate target distances. Verbal reports do not necessarily provide an untransformed measure of perceived distance, but nevertheless, they permit a rough assessment of whether exposure to nonvisual walking causes changes in subsequent indications of target distance that do not involve walking.

\section{Method}

\section{Participants}

Ten males and 10 females consented to participate in exchange for course credit. Their ages ranged from 17 to 20 years (mean $=18.6$ years). All were naive as to the purposes of the study and had normal or corrected-to-normal vision.

\section{Design and Apparatus}

The experiment took place in a well-lit indoor classroom, $3.8 \times$ $10 \mathrm{~m}$. The target was an orange cone, $23 \mathrm{~cm}$ tall. The participants took part in two experimental sessions, separated by an average of 7 days (range: 5-14 days). The sessions were distinguished by whether the participants walked with vision or without vision during a familiarization phase for $3 \mathrm{~min}$ prior to the test phase. Half of the participants walked with vision before Session 1 and without vision before Session 2; the other half received the reverse session order. Assignment to each of these session orders was determined by the time at which the participants arrived, on an alternating basis.

In the test phase, targets could appear at an egocentric distance of $1.5,3.0,4.5$, or $6.0 \mathrm{~m}$ (walking trials) or 1.5, 2.0, 2.5, 3.0, 3.5, $4.0,4.5,5.0,5.5$, or $6.0 \mathrm{~m}$ (verbal trials). Each walking target was presented three times. We used single presentations of multiple distances on the verbal trials in order to minimize the likelihood that the participants would remember specific target locations across sessions. Also, to minimize the possibility that they might create the same verbal responses across sessions because they assumed that the stimuli would be the same, we informed them that they would not see exactly the same target distances in both sessions. To change the set of distances across sessions, first-session trials included two verbal trials with the target placed at $1 \mathrm{~m}$, whereas on second-session trials, there were two verbal trials with the target at $6.5 \mathrm{~m}$. The presentation order of the various combinations of distances and response types (verbal vs. walking) was fully randomized within each session.

\section{Procedure}

The participants who walked without vision during the familiarization phase donned a blindfold and were guided back and forth in the classroom by the experimenter. The participants who walked with vision walked back and forth at a self-determined pace with unrestricted vision. After $3 \mathrm{~min}$, the experimenter guided the participants into the starting position for the test phase. Once in place, the participants donned over-the-ear hearing protectors (overall noise reduction rating: $-20 \mathrm{~dB}$ ), to minimize the influence of auditory cues. On each test phase trial, the participants viewed the target for several seconds and lowered a blindfold. The experimenter then stated which response was to be used on that trial (verbal or walking, which were randomly intermixed). On verbal trials, the participants gave a verbal estimate of the target distance. On walking trials, the experimenter removed the target, and the participants attempted to walk to the target location without vision. The experimenter measured the distance from the starting point to the terminal location and guided the participants back to the starting point without vision and without error feedback.

\section{Results}

We performed repeated measures MANOVAs on the walking and verbal data separately (see Figure 1). Before analysis, we converted the data to signed (constant) errors, expressed as a percentage of the target distance. Walking showed no effect of session order $[F(1,18)=0.02$; $p=.88]$. However, the participants walked significantly farther after they had walked for 3 min without vision in the familiarization phase than after a 3-min walk with vision $\left[F(1,18)=10.87, M S_{\mathrm{e}}=2,423.35, p=.004, \eta_{\mathrm{p}}^{2}=\right.$ .376]. Averaging across distance, the participants tended to undershoot by $3.38 \%$ after a with-vision familiarization phase and tended to overshoot by $4.40 \%$ after a nonvisual familiarization phase. There were no other main effects or significant interactions (all $p \mathrm{~s}>.095$ ).

The verbal data showed only an expected main effect of distance $\left[F(9,162)=9.87, M S_{\mathrm{e}}=5,346.26, p<\right.$ $\left..001, \eta_{\mathrm{p}}^{2}=.354\right]$. There were no main effects of session order $[F(1,18)=1.07, p=.315]$ or familiarization phase $[F(1,18)=0.22, p=.643]$ and no reliable interactions (all $p \mathrm{~s}>.612$ ).
Blind Walking

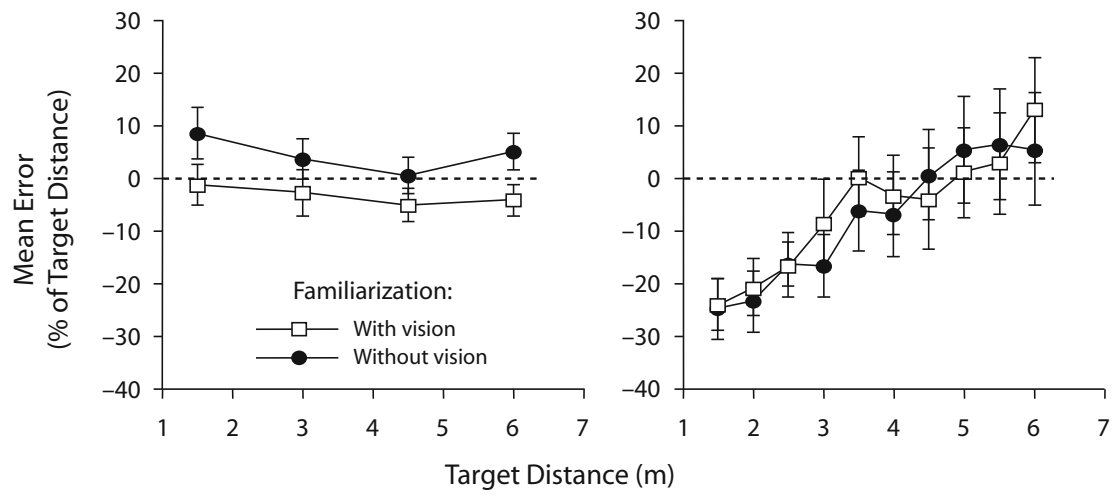

Figure 1. Mean signed errors in Experiment 1 for walking and verbal responses. Error bars denote \pm 1 standard error of the mean. The dashed horizontal lines show the level of accurate responding. 


\section{Discussion}

The participants walked significantly farther after they had been exposed to 3 min of nearly continuous nonvisual walking. On average, the participants walked as much as $56 \mathrm{~cm}$ farther after exposure to nonvisual walking (for a 6.0-m target). This effect was absent in the verbal estimates, suggesting that the change in walked distances was not due to differences in visual perception following visual versus nonvisual walking familiarization. The participants may have differed somewhat in terms of the amount of walking they did immediately before the familiarization phase, but presumably, this would not vary systematically between the with-vision and without-vision familiarization conditions. This argues that the observed increases in blind walking after $3 \mathrm{~min}$ of nonvisual locomotion are not attributable to simple motor adaptation (Anstis, 1995). By itself, Experiment 1 cannot discriminate between changes in walking due to other types of adaptation and changes due to variations in response strategy or participants' confidence in their ability to walk safely without vision. Experiment 2 addressed this issue.

\section{EXPERIMENT 2}

Experiment 1 confirmed that blind-walking responses become recalibrated after exposure to nonvisual locomotion. This recalibration may take place at multiple levels of information processing. In addition to higher level cognitive factors, which we will address shortly, calibration may be affected by adaptation processes operating at a relatively peripheral stage (e.g., motor adaptation) or at a relatively central stage (e.g., adaptation of self-motion perception). Other types of adaptation are also possible (e.g., adaptation based on specific functional goals; Rieser et al., 1995). Running on a treadmill without vision results in both drift aftereffects and significant increases in walked distances, relative to preadaptation baseline performance (Durgin et al., 2005). Given the differing motor (and functional) requirements of running in place versus walking to targets, this suggests that the aftereffects are due to adaptation at a relatively central stage and that the adaptation is not strictly goal specific. In addition, when walked distances increase in a blind-walking task after treadmill locomotion, this is apparently achieved by increasing the number of paces, rather than by lengthening each stride (Durgin et al., 2005; Rieser et al., 1995). This also argues against adaptation at a relatively peripheral, motoric level. Thus, measuring signed errors and stride length in blind walking, along with comparing marching-in-place drifting before and after blind walking, promises to provide insights into the type of adaptation that might occur as participants gain exposure to nonvisual locomotion.

To address these issues, the participants in Experiment 2 performed 37 successive trials in a blind-walking task. We examined walked distance and pace length as a function of the cumulative exposure to nonvisual walking (as indexed by successive trial numbers). We also assessed the amount of unintentional forward drift as the participants attempted to march in place without vision. If there was not only a ten- dency toward increasing walked distances as trials progress, but also increased forward drift after the 37 blind-walking trials, this would suggest that at least some of the increases in walked distance were due to perceptuomotor (as opposed to purely motor) adaptation. This conclusion would be corroborated if increases in walked distance were achieved without corresponding increases in pace length.

Recalibration of walking might also arise due to changes in higher level cognitive factors as the experiment unfolded, such as increases in the participants' familiarity with the task. Familiarity may include such subjective factors as participants' feelings of confidence that they are able to walk safely without vision and confidence in executing the procedural aspects of the task. This is more difficult to assess than adaptation-related changes, but in Experiment 2, we examined this possibility by measuring the mean walking velocity on each trial. We will interpret increases in self-selected velocity as an operationalization of increased confidence or familiarity with the task. Is there any way to disentangle the possible effects of adaptation from familiarity? We attempted to do so in the following manner. After the blind-walking trials, we gave the participants several minutes' exposure to walking to targets under visual control. We predicted that if the anticipated changes in walking performance as the experiment progresses were due primarily to adaptation, reexposure to eyes-open walking should return walking calibration to a state closer to that in the beginning of the experiment, resulting in shorter walking responses. By contrast, a short reexposure to eyes-open walking presumably would not decrease familiarity with blind walking. If increasing task familiarity strongly contributes to progressive changes in walking errors, one would not expect performance to differ before and after eyes-open practice.

Finally, walked distances might increase due to changes in the participants' response strategy (another higher level cognitive factor). To assess this, we asked the participants to complete a questionnaire targeting their strategies and subjective feelings of confidence in walking throughout the experiment.

\section{Method}

\section{Participants}

Fourteen males and 13 females participated in exchange for course credit. ${ }^{1}$ Their ages ranged from 18 to 21 years (average = 19.3 years). All were naive as to the purposes of the study and had normal or corrected-to-normal vision.

\section{Design and Apparatus}

The experiment took place outdoors in a flat, grassy field $(70 \times$ $140 \mathrm{~m}$ ). There were several trial types, which always occurred in the following order (see also Table 1): marching in place 1, blind walking 1 , marching in place 2 , eyes-open walking, marching in place 3 , blind walking 2 , and questionnaire. Response time, straight-line walked distance, and number of paces were recorded on all blindwalking trials. Paces and walking duration were not analyzed directly but were used to calculate mean velocity and mean pace length on each trial. Target distances on blind-walking trials were indicated by an orange cone ( $23 \mathrm{~cm}$ tall) placed on the ground, with possible target distances being $4,8,10,12$, and $16 \mathrm{~m}$. The 10 -m target was presented as a probe distance on the very first blind walking 1 trial and then on every 9 th trial thereafter for all the participants (five times total). 
Table 1

Sequence of Trial Types in Experiment 2

\begin{tabular}{lcc}
\hline \multicolumn{1}{c}{ Trial Type } & $\begin{array}{c}\text { Number of } \\
\text { Trials }\end{array}$ & \multicolumn{1}{c}{ Targets } \\
\hline $\begin{array}{lcc}\text { 1. Marching in place, Phase 1 } \\
\text { 2. Blind walking 1 }\end{array}$ & 1 & no target \\
& 37 & $\begin{array}{c}10 \text {-m probe (five times) } \\
4,8,12,16 \mathrm{~m}(\text { eight } \\
\text { times apiece) }\end{array}$ \\
3. Marching in place, Phase 2 & 1 & no target \\
4. Eyes-open practice & $(10 \mathrm{~min})$ & \\
5. Marching in place, Phase 3 & 1 & no target \\
6. Blind walking 2 & 5 & 10 -m probe; 4, 8, 12, \\
& & 16 m (once apiece) \\
\hline
\end{tabular}

aA probe target was presented on Trials 1, 10, 19, 28, and 37. The other four target distances were presented in four blocks of eight trials each. Each block presented the four target distances twice apiece in random order.

Between each of these probe trials, there was a block of 8 walking trials, consisting of the four other target distances presented twice apiece in random order. Thus, the blind walking 1 trials were divided into four sequential blocks of 8 trials each, separated by the probe trials (37 trials total). This arrangement allowed us to use the probe trials to track systematic changes in performance for one particular target while controlling for the amount of intervening exposure to nonvisual walking. Trials involving the other targets provided a somewhat coarser measure of time-varying systematic changes but allowed an assessment of the extent to which these changes depended on target distance. On the blind walking 2 trials, the first trial used the $10-\mathrm{m}$ probe target distance for all the participants. The remaining 4 trials used the other four possible distances in random order.

\section{Procedure}

Marching in place 1. All the marching-in-place trials took place on a sidewalk adjoining the grassy field. The participants were instructed to lift their knees high and to attempt to remain in the same location when marching. In addition to a blindfold, all the participants wore hearing protectors to minimize the influence of auditory cues (Durgin \& Pelah, 1999). They then attempted to march in place for $20 \mathrm{sec}$. An experimenter timed the duration, using a stopwatch. Any distance traveled during this period was recorded using a tape measure. The participants remained blindfolded and were guided without vision to the starting point of the blind-walking trials, without obtaining any error feedback.

Blind walking 1 trials. While wearing hearing protectors, the participants viewed the target cone binocularly and then attempted to walk to its location while blindfolded, using their normal walking pace. Response time was measured by an experimenter using a stopwatch; the stopwatch was started when the participants first lifted their leg to begin walking and was stopped when they stopped walking. After stopping, they remained blindfolded while their straightline response distance was recorded. The number of paces was also recorded. The participants were then guided back to the starting

Table 2

Questionnaire With Response Frequencies Used in Experiment 2

Questions and Response Alternatives

Frequency $(\%)^{\mathrm{a}}$

1. Which of the following best describes your impression of how close you came to the target in the very FIRST trial of the experiment?

I feel like I stopped 2 feet or more short of the target

65

I feel like I stopped 2 feet or more past the target

I feel like I stopped within 2 feet of the target

Other

2. Which of the following best describes your impression of how close you came to the target in the very LAST trial of the experiment?

I feel like I stopped 2 feet or more short of the target

I feel like I stopped 2 feet or more past the target

I feel like I stopped within 2 feet of the target

Other

3. If you feel that you changed how far you walked to the targets as the experiment progressed, which

of the following best describes why you changed? (please choose only ONE)

Began to concentrate more

Began to concentrate less

Concentrated the same amount, but on a different aspect of the task

Became more concerned about stumbling

Became less concerned about stumbling

Became more comfortable with walking blindfolded, but was never very concerned about stumbling

Became less comfortable with walking blindfolded, but was never very concerned about stumbling

Felt that your first few responses were inaccurate, so you tried to compensate

Other

4. Which of the following best describes your overall level of concern about whether you might stumble while walking blindfolded?

More concerned in the last 3 trials than in the first 3 (increasing concern)

Less concerned in the last 3 trials than in the first 3 (decreasing concern)

Not very concerned, during any part of the experiment

Concerned, throughout the experiment

Other

5. Which of the following best describes the effect of your concern about stumbling?

This concern did not influence how far I walked

When I was less concerned about stumbling, I walked farther

When I was more concerned about stumbling, I walked farther

Other

aResponse frequencies are expressed as a percentage of the total number of responses for each question $(N=27)$. Response

frequencies may not sum to $100 \%$ on particular questions, due to rounding.

12

22

22

22

56

30

26

0

30

11

4

22

70

78

19

4

\begin{tabular}{l}
4 \\
4 \\
0 \\
4 \\
4 \\
\hline
\end{tabular}


point without error feedback. After 37 trials, they were guided without vision to the nearby sidewalk to complete the second marchingin-place trial. Including the outbound and inbound walking paths, the total distance walked without vision on the blind walking 1 trials was approximately $740 \mathrm{~m}$.

Marching in place 2. The methods were the same as those used for the first marching trials.

Eyes-open practice. These trials were intended to provide exposure to visually guided walking; no data were collected. Stimulus locations were indicated by six orange rods $(0.8 \mathrm{~cm}$ diameter $X$ $122 \mathrm{~cm}$ tall) stuck vertically into the ground in a rectangular configuration $(4 \times 16 \mathrm{~m}$, with two rods placed midway on the long sides). A common object name was written on a folded sheet of paper attached to the top of each rod (car, tomato, football, hammer, dog, chair). The participants learned the object names associated with each of the six stimulus locations by walking among the locations with eyes open. After several minutes of this learning phase, an experimenter read off sequences of three, four, five, and six object names, and the participants attempted to walk with vision to the named target locations, in the order named. Eyes-open practice was terminated after $10 \mathrm{~min}$.

Marching in place 3. The methods were the same as those used for the other marching trials.

Blind walking 2. Five more blind-walking trials were conducted using the same methods as in blind walking 1 .

Questionnaire. After the last blind-walking trial, the participants completed the questionnaire shown in Table 2.

\section{Data Analyses}

Analyses of most trial types will be described in more detail in the Results section, but here we introduce the blind walking 1 analyses, due to their complexity. Primarily, these analyses involved separate repeated measures MANOVAs on the walking distances, mean velocities, and mean pace lengths. These tests were further divided into analyses of probe target trials (10-m target distance) and other target trials (4-, 8-, 12-, and 16-m distances). The probe target analyses included probe repetition number as a within-subjects variable (with repetition numbers $1-5$, corresponding to trials $1,10,19,28$, and 37 , respectively). Analyses of the other targets included trial quarter as a within-subjects variable, with responses to each target distance being analyzed as they appeared in the first, second, third, and fourth quarters of the trials. Our primary prediction concerned the analysis of walked distance, in which we expected main effects of probe repetition number and trial quarter. If there were a main effect of trial quarter in the MANOVAs, planned comparisons between quarters could provide insight into the temporal structure of the changes. The MANOVAs also allowed us to investigate these changes as a function of target distance. We were also interested in possible increases in mean velocity and pace length, since these might indicate roles for task familiarity and motor adaptation, respectively, in blind-walking performance.

\section{Results}

\section{Marching in Place}

The mean unintentional forward drift in marching-inplace trials is shown in Figure 2. We performed a repeated measures ANOVA on these trials, with phase varied within subjects (Phase 1, just prior to Blind-Walking Trial 1; Phase 2, just after Blind-Walking Trial 37; Phase 3, after eyes-open practice). This analysis showed a main effect of phase $\left[F(2,52)=4.01, M S_{\mathrm{e}}=0.36, p=.024, \eta_{\mathrm{p}}^{2}=.138\right]$. Planned contrasts showed that the participants drifted significantly more in Phase 2 than in Phase 1, and drifted significantly less in Phase 3 than in Phase 2. The amount of drift between Phase 3 and Phase 1 did not differ significantly. The mean increase in drift in Phase 2, relative to Phase 1,

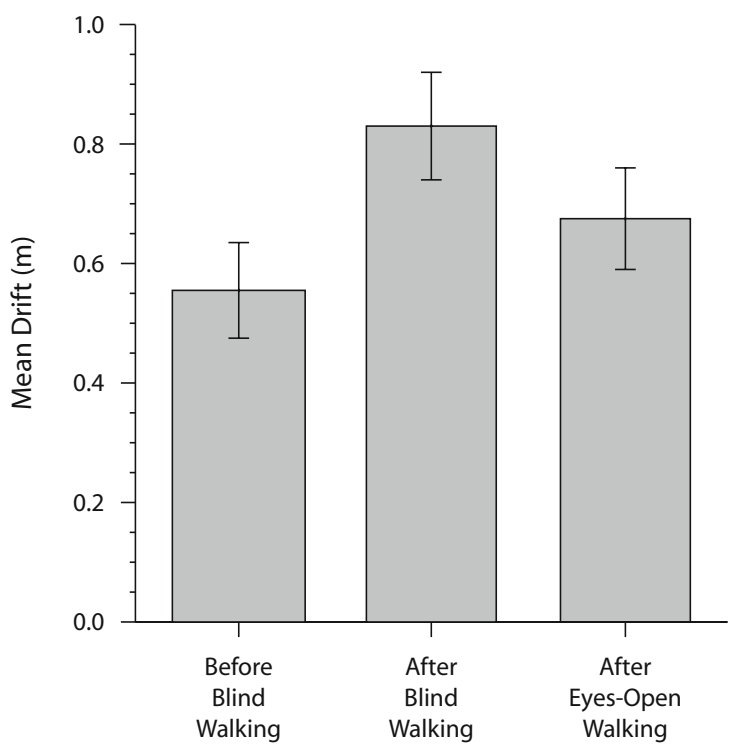

Figure 2. Mean distances that the participants unintentionally drifted forward when attempting to march in place without vision (Experiment 2). Drift at three time points is shown: "Before Blind Walking" is just prior to Trial 1 in the main block of blindwalking trials; "After Blind Walking" is just after Blind-Walking Trial 37; "After Eyes-Open Walking" is immediately after 10 min of visually guided walking. Error bars show \pm 1 standard error of the mean.

was $+0.27 \mathrm{~m}$. The mean decrease in drift in Phase 3 , relative to Phase 2, was $-0.15 \mathrm{~m}$ (see Figure 2). There was some drift (averaging $+0.55 \mathrm{~m}$ ) even during the baseline test. Although baseline drift is not uncommon (e.g., Anstis, 1995; Durgin \& Pelah, 1999), it is poorly understood. It may be at least partly an aftereffect of the state of motor adaptation elicited by normal, visually guided locomotion.

\section{Blind Walking: Walked Distance}

Overall accuracy. There was a tendency for walked distances to increase across trials. Signed errors, expressed as a percentage of the target distance and averaged over participant and distance, are shown in Figure 3 (top panel) as a function of trial number. To obtain an estimate of the total mean change in accuracy across the experiment, we fit a straight line to these data, using the least-squares criterion. The best-fitting line intersected Trial 1 at $-2.44 \%$ and intersected Trial 37 at $+6.89 \%$, for a difference of $9.32 \%$.

Probe target. A repeated measures ANOVA on the walked distance for probe trials, with repetition number as the within-subjects factor, showed no main effect of probe repetition number $[F(4,104)=1.55, p=.192]$. Although this result is contrary to our predictions, it likely reflects the limited statistical power of the probe target analysis. We will return to this issue in the Discussion section. The mean performance for Repetitions $1-5$ on 10 -m probe trials was $9.8,10.1,10.4,10.3$, and $10.6 \mathrm{~m}$, respectively.

Other targets. This repeated measures MANOVA (see Table 3) showed a main effect of quarter, confirming that there was a tendency for walking responses to indi- 

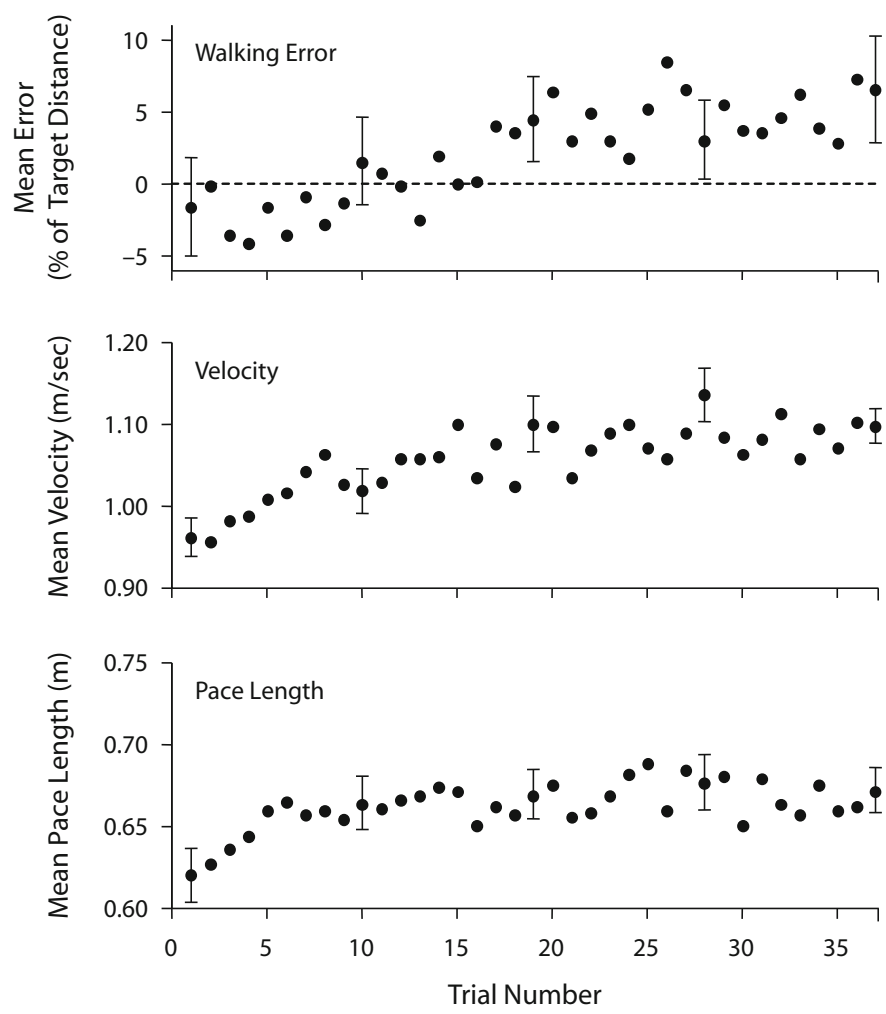

Figure 3. Blind-walking performance in Experiment 2 as a function of trial number. Data are collapsed over target distance and participant on each trial. Top panel: Signed walking errors, expressed as a percentage of the target distance; the dashed horizontal line shows the level of accurate responding. Middle panel: Mean walking velocity in meters per second. Bottom panel: Mean pace length in meters. Trials $1,10,19,28$, and 37 were probe trials and, therefore, show data from only a single target distance $(10 \mathrm{~m})$; error bars on these trials show \pm 1 one standard error of the mean. For the other trials, individual participants were generally presented with different distances $(4,8,12$, or $16 \mathrm{~m})$. Across all participants, there was virtually no correlation between physical target distance and trial number $(r=-.03)$, but, due to the random ordering of conditions for each participant, the average physical target distance across participants varied somewhat from trial to trial. The data shown in the figure are likely perturbed by these variations to some extent.

vidual targets to increase as the experiment progressed (Table 4). Pairwise planned contrasts (two-tailed; $\alpha=$ .05 ) showed that the difference between quarters was generally reliable, except for that between Quarters 3 and $4(p=.928)$ and a marginal difference between Quarters 1 and $2(p=.056)$. There was a tendency for errors to increase with distance (Table 5). Planned contrasts showed that this effect was driven by significant differences between the 16- $\mathrm{m}$ target and the 4- and 8-m targets ( $p \mathrm{~s}=.032$ and .005 , respectively; all other $p \mathrm{~s}>$ $.08)$. There was no quarter $\times$ distance interaction.

\section{Blind Walking: Velocity}

Mean walking velocity, averaged over participant and target distance, is shown in Figure 3 (middle panel) as a function of trial number.
Probe target. The repeated measures ANOVA on mean walking velocity for probe trials, with repetition number as the within-subjects factor, revealed a main effect of repetition number $\left[F(4,104)=15.83, M S_{\mathrm{e}}=0.16, p<.001\right.$, $\left.\eta_{\mathrm{p}}^{2}=.379\right]$

Other targets. The repeated measures MANOVA for other targets demonstrated a main effect of both quarter and distance (see Table 3). As in the probe target analysis, velocities tended to increase as the experiment progressed (Table 4), although planned contrasts showed that only the increases between Quarters 1 and 2 were significant, with no further reliable changes after that. Velocities also tended to increase with target distance (Table 5). Planned contrasts showed that velocities differed between all pairs of distances except 12 and $16 \mathrm{~m}$. There was no quarter $\times$ distance interaction. 
Table 3

Results of Repeated Measures MANOVAS for Experiment 2 (Nonprobe Targets)

\begin{tabular}{llrrcc}
\hline \multicolumn{1}{c}{$d f$} & \multicolumn{1}{c}{$M S_{\mathrm{e}}$} & $F$ & $p$ & $\eta_{\mathrm{p}}^{2}$ \\
\hline Walked Distance & & & & & \\
$\quad$ Distance & 3,78 & 511.16 & 3.21 & $.027^{*}$ & .110 \\
$\quad$ Quarter & 3,78 & $1,094.11$ & 8.91 & $<.001^{* *}$ & .255 \\
$\quad$ Distance $\times$ quarter & 9,234 & 31.00 & 0.69 & .715 & .026 \\
Velocity & & & & & \\
$\quad$ Distance & 3,78 & 0.50 & 57.46 & $<.001^{* *}$ & .688 \\
$\quad$ Quarter & 3,78 & 0.11 & 7.94 & $<.001^{* *}$ & .234 \\
$\quad$ Distance $\times$ quarter & 9,234 & $<0.01$ & 0.87 & .544 & .033 \\
Pace Length & & & & & \\
$\quad$ Distance & 3,78 & 0.02 & 7.29 & $<.001^{* *}$ & .219 \\
Quarter & 3,78 & $<0.01$ & 3.21 & $.027^{*}$ & .110 \\
$\quad$ Distance $\times$ quarter & 9,234 & $<0.01$ & 0.60 & .795 & .023 \\
Within-Subjects $S D$ & & & & & \\
$\quad$ Distance & 3,75 & 20.51 & 35.36 & $<.001^{* *}$ & .576 \\
Quarter & 3,75 & 1.87 & 3.50 & $.019^{*}$ & .119 \\
$\quad$ Distance $\times$ quarter & 9,225 & 0.72 & 1.32 & .226 & .048 \\
\hline
\end{tabular}

Note $-d f$, degrees of freedom; $M S_{\mathrm{e}}$, mean squared error; $S D$, standard deviation. ${ }^{*}$ Significant at $\alpha=.05 .{ }^{* *}$ Significant at $\alpha=.01$.

Table 4

Mean Walking Parameters in Experiment 2 As a Function of Experiment Quarter (With Between-Subjects Standard Errors of the Means; $N=27$ )

\begin{tabular}{|c|c|c|c|c|c|c|c|c|}
\hline \multirow[b]{3}{*}{ Quarter } & \multicolumn{8}{|c|}{ Walking Parameter } \\
\hline & \multicolumn{2}{|c|}{$\begin{array}{c}\text { Signed Error } \\
(\%)\end{array}$} & \multicolumn{2}{|c|}{$\begin{array}{l}\text { Velocity } \\
(\mathrm{m} / \mathrm{sec})\end{array}$} & \multicolumn{2}{|c|}{$\begin{array}{c}\text { Pace } \\
\text { Length }(\mathrm{m}) \\
\end{array}$} & \multicolumn{2}{|c|}{$S D(\mathrm{~m})$} \\
\hline & $M$ & SEM & $M$ & $\overline{S E M}$ & $M$ & SEM & $M$ & $S E M$ \\
\hline First & -1.89 & 1.29 & 1.01 & 0.02 & 0.65 & 0.01 & 0.89 & 0.08 \\
\hline Second & 1.03 & 1.31 & 1.05 & 0.02 & 0.66 & 0.01 & 0.82 & 0.08 \\
\hline Third & 4.60 & 1.37 & 1.07 & 0.02 & 0.67 & 0.01 & 0.75 & 0.08 \\
\hline Fourth & 4.74 & 1.47 & 1.08 & 0.02 & 0.67 & 0.01 & 0.58 & 0.05 \\
\hline
\end{tabular}

Note-Signed error is expressed as percentage of target distance. $S D$, within-subjects standard deviation.

Table 5

Mean Walking Parameters in Experiment 2 As a Function of Target Distance (With Between-Subjects Standard Errors of the Means; $N=27$ )

\begin{tabular}{|c|c|c|c|c|c|c|c|c|}
\hline \multirow{2}{*}{$\begin{array}{c}\text { Target } \\
\text { Distance (m) }\end{array}$} & \multicolumn{2}{|c|}{$\begin{array}{c}\text { Signed Error } \\
(\%)\end{array}$} & \multicolumn{2}{|c|}{$\begin{array}{l}\text { Velocity } \\
(\mathrm{m} / \mathrm{sec})\end{array}$} & \multicolumn{2}{|c|}{$\begin{array}{c}\text { Pace } \\
\text { Length }(\mathrm{m}) \\
\end{array}$} & \multicolumn{2}{|c|}{$S D(\mathrm{~m})$} \\
\hline & $M$ & $\overline{S E M}$ & $M$ & $\overline{S E M}$ & $M$ & $\overline{S E M}$ & $M$ & $\overline{S E M}$ \\
\hline 4 & 1.06 & 1.45 & 0.96 & 0.0 & 0.64 & 0.01 & 0.26 & 0.02 \\
\hline 8 & -0.20 & 1.37 & 1.05 & 0.02 & 0.66 & 0.01 & 0.57 & 0.05 \\
\hline 12 & 2.83 & 1.38 & 1.09 & 0.02 & 0.67 & 0.01 & 0.93 & 0.10 \\
\hline 16 & 4.80 & 1.30 & 1.11 & 0.02 & 0.68 & 0.01 & 1.26 & 0.10 \\
\hline
\end{tabular}

Note-Signed error is expressed as percentage of target distance. $S D$, within-subjects standard deviation.

\section{Blind Walking: Pace Length}

Mean pace length, averaged over participant and target distance, is shown in Figure 3 (bottom panel) as a function of trial number.

Probe target. The repeated measures ANOVA on mean pace length for probe trials, with repetition number as the within-subjects factor, showed a main effect of repetition number $\left[F(4,104)=8.97, M S_{\mathrm{e}}=0.01, p<.001, \eta_{\mathrm{p}}^{2}=\right.$ .257]. Pace lengths tended to increase across repetition, with mean pace lengths for Probe Target Repetitions 1-5 being $0.62,0.66,0.67,0.68$, and $0.67 \mathrm{~m}$, respectively.
Other targets. The repeated measures MANOVA for other targets demonstrated main effects of both quarter and distance (see Table 3). Planned contrasts showed that mean pace lengths for the 4-m target were reliably shorter than those for the other distances (by 3-4 cm), whereas mean pace lengths for the $8-\mathrm{m}$ target were reliably shorter (by $1-2 \mathrm{~cm}$ ) than those for the 12-m target. Mean pace lengths for the other targets did not differ among themselves. Planned contrasts also showed that pace lengths for the first quarter were reliably shorter than those for the other quarters (by 1-2 cm), which did not differ among themselves. There was no quarter $\times$ distance interaction.

\section{Blind Walking: Within-Subjects \\ Standard Deviations}

Other targets. To obtain a coarse measure of the within-subjects response variability as the experiment progressed, we calculated within-subjects standard deviations $(S D s)$ across two measurements per target distance in each quarter of the experiment (see Figure 4). ${ }^{2} \mathrm{~A}$ repeated measures MANOVA on the within-subjects $S D$ s of the walked distances showed a main effect of quarter, with $S D$ s tending to decrease across quarters (Tables 3 and 4). Planned contrasts showed that $S D$ s in the fourth quarter were reliably lower than those in Quarters 1 and $2(p$ s $=$ .005 and .019 , respectively), with no other significant differences (all other $p \mathrm{~s}>.126$ ). There was also a main effect of distance, with mean $S D$ s increasing with target distance (Table 5). Planned comparisons showed reliable differences between all pairs of distances (all $p \mathrm{~s}<.030$ ). There was no quarter $\times$ distance interaction.

\section{Effect of Eyes-Open Practice on Blind Walking}

We compared walking responses for the final appearance of each target distance in the last quarter with walking responses immediately after exposure to $10 \mathrm{~min}$ of visually guided walking. We compared probe target responses before versus after the practice session, using two-tailed $t$ tests $(d f=26 ; \alpha=.05)$. For other target walked distances, we performed a repeated measures MANCOVA with phase (pre- vs. postpractice) and target distance as within-subjects factors. To test whether there was a significant relationship between the amount of change in walked distances and the amount of change in drift (pre- vs. postpractice), we calculated drift difference scores (postpractice minus prepractice) and included these scores as a covariate in the model. Other target velocity and pace length data were analyzed using repeated measures MANOVAs with phase and target distance as within-subjects factors. All analyses of other target data showed reliable main effects of distance that mirrored those in the main experiment and no significant distance $\times$ phase interactions, so we will not describe these effects in detail here.

Walked distances (probe targets). The $t$ test showed that the participants walked significantly less to the $10-\mathrm{m}$ probe target after eyes-open practice than just prior to this practice $(p=.037)$. The average signed error was $+0.66 \mathrm{~m}$ prior to eyes-open practice and $-0.02 \mathrm{~m}$ after. 


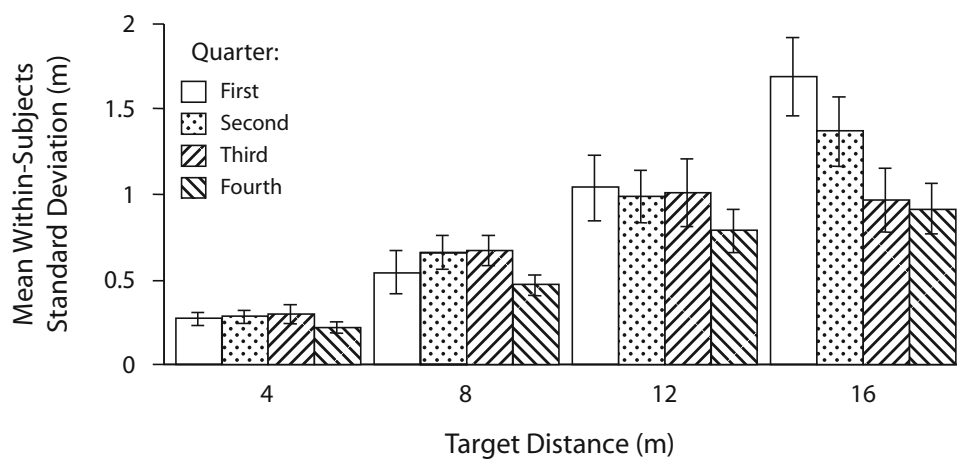
Figure 4. Mean signed walking error in Experiment 2 as a function of target distance
and experiment quarter. Positive errors denote overshooting. Error bars show \pm 1
standard error of the mean.

Walked distances (other targets). There was a nonsignificant trend for an effect of phase $[F(1,26)=2.97$, $\left.M S_{\mathrm{e}}=342.49, p=.097, \eta_{\mathrm{p}}^{2}=.106\right]$. The mean signed error before eyes-open practice was $+4.23 \%$ of the target distance and $+6.68 \%$ afterward. Change in drift from preto postpractice did not account for a significant portion of the variance in the model.

Velocity (probe targets). There was a marginal effect of phase $(p=.070)$, with mean velocities before and after eyes-open practice being 1.10 and $1.15 \mathrm{~m} / \mathrm{sec}$, respectively.

Velocity (other targets). There was a main effect of phase $\left[F(1,26)=5.66, M S_{\mathrm{e}}=0.05, p=.025, \eta_{\mathrm{p}}^{2}=.179\right]$, with mean velocities before and after eyes-open practice being 1.09 and $1.12 \mathrm{~m} / \mathrm{sec}$, respectively.

Pace length (probe targets). There was no effect of phase ( $p=.399)$; pace lengths before and after eyes-open practice were 0.67 and $0.68 \mathrm{~m}$, respectively.

Pace length (other targets). This ANOVA showed a very small but reliable effect of phase $\left[F(1,25)=5.02,{ }^{3}\right.$ $\left.M S_{\mathrm{e}}=0.01, p=.034, \eta_{\mathrm{p}}^{2}=.167\right]$, with mean pace lengths before and after eyes-open practice being 0.66 and $0.67 \mathrm{~m}$, respectively.

\section{Questionnaire}

The participants tended to report believing themselves to be walking farther at the end of the experiment than at the beginning (see Table 2). Although this impression generally matched the pattern of their actual performance, they were never given any error feedback. Thus, their assumed accuracy may not bear any relation to factors that actually affected their performance. On the other hand, it is possible that they were aware of conscious strategy changes or other internal changes that may, indeed, have affected their performance. Supporting this notion, a good many participants $(56 \%)$ cited changes in concentration as the source of their variations in assumed accuracy, whereas others $(30 \%)$ cited increasing comfort with the walking response. Interestingly, a strong majority $(70 \%)$ reported not being very concerned about stumbling during any part of the experiment, and most (78\%) felt that concerns about stumbling did not influence their walking.

\section{Correlating Walking Errors With Velocity and Pace Length}

Although participants might become more familiar with the task across trials and begin walking faster as a result, in principle, these changes could occur without impacting walking error at all. Nevertheless, if one source of the gradual changes in walking error is indeed increasing familiarity with the task, one might expect increases in familiarity (operationalized here by increases in mean walking velocity) to account for some of the variance in walking errors. To address this, we converted the walked distances into signed errors. We then correlated these errors (for all participants and all targets) with walking velocity in the first, second, third, and fourth quartiles of the experiment. All four $r^{2}$ coefficients were extremely low $(<.003)$ and did not differ significantly from zero [all $F \mathrm{~s}(1,242)<1.85$, all $p \mathrm{~s}>.05$ ].

If increases in walked distance are associated with increases in pace lengths, this could indicate that relatively peripheral (i.e., motor) adaptation processes are responsible (Durgin et al., 2005). To test this, we correlated signed walking errors with mean pace lengths for each quartile of the experiment, and again all four $r^{2}$ coefficients were extremely low $(<.01)$ and did not differ significantly from zero [all $F_{\mathrm{s}}(1,242)<3.59$, all $p \mathrm{~s}>.05$ ]. These findings suggest that changes in velocity and pace length accounted for very little of the variance in walking error at any stage of the experiment.

\section{Discussion}

There was a drift aftereffect (averaging $0.27 \mathrm{~m}$ over $20 \mathrm{sec}$ ) after exposure to approximately $720 \mathrm{~m}$ of nonvisual walking on solid ground. Proffitt et al. (2003) found a somewhat greater difference in drift between pre- and postadaptation (averaging $0.60 \mathrm{~m}$ over $20 \mathrm{sec}$ ) following 3 min of treadmill walking while participants viewed an unchanging scene in a helmet-mounted display. The increased drift may have occurred because their participants walked at a higher velocity $(1.34 \mathrm{~m} / \mathrm{sec}$, vs. our observed mean of $1.07 \mathrm{~m} / \mathrm{sec}$ ). In our study, this drift aftereffect was coupled with a tendency to walk farther for each target as the experiment progressed. The progressive increases in walking 
were most evident in the analysis of the 4-, 8-, 12-, and 16-m target distances, in which walked distances increased significantly as a function of experiment quarter. Although the mean walked distance increased somewhat for probe trials, this difference was not significant. The apparent difference in results between probe trials and other target trials may be due to lower statistical power in the probe trial analysis. The number of probe trials included in the design was somewhat arbitrary; we chose five in order to allow a relatively fine-grained assessment of changes as the experiment progressed. One drawback of this choice is that as the number of probe trials increases, there is a corresponding increase in the statistical power required in order to detect significant differences across all probe trials. Also, the probe target analysis was based on a single measurement per participant for each probe repetition number. These factors decrease the effective statistical power of the probe target analysis and, no doubt, contribute to the apparent differences in results, relative to the other target analysis.

Interestingly, Rieser et al. (1995) found no differences in blind-walking responses before and after $10 \mathrm{~min}$ of nonvisual treadmill walking. Differences in walking speed are unlikely to be the full explanation for the apparent discrepancy, because our walking speeds during adaptation were somewhat slower than those of Rieser et al. (1995), averaging 1.07 versus $1.11 \mathrm{~m} / \mathrm{sec}$, respectively. In fact, to our knowledge, ours is the slowest mean velocity that has been shown to result in locomotor aftereffects. Differences in statistical power may be partly responsible (our $N=27$, vs. $N=6$ in Rieser et al., 1995).

One possible cognitive factor underlying the gradual increase in walking comes from increasing familiarity with the task. Walking velocity increased considerably (by $16 \mathrm{~cm} / \mathrm{sec}$, on average). Response variability tended to decrease, as indicated by the within-subjects $S D$ s. These results collectively support the notion that the participants were becoming more confident, and a good many participants explicitly reported an increasing level of comfort with the task. We found little evidence, however, that increases in familiarity were responsible for increases in walked distance: Mean walking velocity accounted for virtually no variance in walking errors. Interestingly, increased familiarity was generally not associated with increased feelings of safety. A large majority of the participants reported not being concerned about stumbling during any part of the experiment. Taken at face value, these self-reports belie the notion that practice walking without vision increases participants' subjective feeling of safety, at least in terms of their ability to walk without stumbling. Some safety-related changes in walked distance might nevertheless occur without awareness, however.

Some of the progressive overshooting might also be due to changes in response strategy, since $77 \%$ of the participants reported changes in concentration or explicit attempts to correct for perceived inaccuracies early in the experiment. The relative weighting of changes due to adaptation versus differing response strategies is unclear. However, given the significant increase in drift after 37 trials of blind walking and the decrease in drift after $10 \mathrm{~min}$ of eyes-open practice (see below), this strongly suggests that at least part of the tendency to progressively overshoot in blind-walking trials was due to perceptuomotor adaptation. Given the differing functional goals of these two tasks (marching in place vs. walking to a previously viewed target), this suggests that the adaptation is not goal specific.

The eyes-open practice and the subsequent drift and walking trials were intended to provide a means of discriminating between adaptation and familiarity effects. Velocities and pace lengths remained approximately the same after eyes-open practice, albeit with some slight increases. This suggests that participants remained confident about their ability to walk while blindfolded. The participants walked a significantly shorter distance to the $10-\mathrm{m}$ probe target after eyes-open practice and also exhibited less unintentional forward drifting when attempting to march in place. These data suggest that reexposure to visually guided walking returned the coupling between visual and nonvisual self-motion signals to a state similar to that at the beginning of the study. However, there was no effect of eyes-open practice in the walked distance analysis involving the full range of targets. Although this could support the notion that the gradual changes in walking are entirely due to familiarity and/or response strategy, the correspondence between the drift data and the probe target analysis for walking argues that adaptation does, indeed, play a significant, although perhaps not exclusive, role. Durgin et al. (2005) have shown that blind walking is somewhat less susceptible to adaptation-related effects than is forward drifting - specifically, in terms of its sensitivity to the duration of adaptation. The authors speculate that walking calibration may exhibit more "inertia" than does drift, perhaps because walking is more common in everyday life than is marching in place. This could reconcile the apparent discrepancy between the changes in drift and probe target trials, on the one hand, and the lack of changes in walking to the remaining targets, on the other hand. Another possibility is that, by the end of the experiment, our participants had consciously or unconsciously encoded the approximate amount of time it took to walk to each target. Then, after the eyes-open practice, they were biased toward reproducing these walking times during the subsequent blind-walking trials.

The lack of a quarter $\times$ distance interaction in the walked distance errors indicates that there was no target-specific adaptation (Ellard \& Shaughnessy, 2003): The changes across quarter remained roughly proportional to target distance for all the targets. This gain-change recalibration is consistent with both motor adaptation and adaptation of self-motion perception. However, the pace length analyses showed that the increases in walked distance across quarters cannot be explained by increases in pace lengths, because walked distances increased throughout the experiment, whereas pace lengths increased by only a small amount early in the experiment $(1-2 \mathrm{~cm})$ and then remained roughly constant. This argues against adaptation at the purely motor level (Durgin et al., 2005) and supports the view that the gain changes across experiment quarters were due to adaptation of self-motion perception. A potential counterargument to this interpretation, however, comes from the general ineffectiveness of eyes-open practice at reducing overshooting 
in walked distances. This finding is more easily explained under the assumption of motor adaptation than of perceptuomotor adaptation. Taken together, Experiment 2 shows that perceptuomotor adaptation accounts for at least some of the gradual increases in blind-walking responses across trials, but motor adaptation may also play a role.

The fact that the gain change across quarters was more or less constant for all the target distances has another implication. Some researchers have suggested that visually perceived egocentric distance is a compressively nonlinear function of physical distance, even in well-lit, outdoor viewing conditions (Gilinsky, 1951). The progressive adaptation we observed in Experiment 2 is approximately linear with target distance and, therefore, does not appear to be developing in a way that would tend to offset a nonlinearity in perceived distance (see also Loomis \& Philbeck, 2008).

Mittelstaedt and Mittelstaedt (2001) found that accuracy in a visually directed walking task varied with walking velocity; participants walked accurately if they selected their own velocity but produced shorter walking responses if they were constrained to walk faster than their self-selected velocity (see also Schwartz, 1999). Although we found that the participants tended to walk faster as the experiment progressed, this was associated with longer walking responses. Our participants selected their own velocity, so apparently the linkage between velocity and blind-walking responses is critically dependent on whether or not participants walk at a self-selected speed.

As a final observation, we will note that just after the participants terminated each walking response, there was a short pause while the experimenters measured and recorded the walked distance; just after the participants returned to the starting position, there was another short pause while the experimenters prepared for the next trial. As the experimenters became more practiced, these pauses shortened from tens of seconds down to 1 or $2 \mathrm{sec}$. We noticed that the participants appeared more likely to exhibit progressive overshooting if they were tested later in the group. Splitting the group approximately in half, trial number accounted for $10 \%$ of the variance in signed percent errors for the first half of the participants and 79\% for the last half. (The squared correlation for the group as a whole was .69; see Figure 3, top panel, for a scatterplot of the data underlying this correlation.) The methods used were otherwise similar across participants, so interrupting the exposure to nonvisual walking with periods of standing may play a role in attenuating recalibration effects.

\section{GENERAL DISCUSSION}

These experiments show that walked distances in a blind-walking task tended to increase as exposure to nonvisual walking accumulated during an experimental session. We considered several sources of these systematic changes in blind-walking responses. The verbal responses in Experiment 1 suggested that changes in walked distance following nonvisual locomotion were not due to changes in visually perceived target distance. Several participants in Experiment 2 reported changes in concentration and explicit attempts to correct for perceived errors, so variations in response strategy may play some role. By contrast, although most participants reported becoming increasingly comfortable with the task, few endorsed the idea that their concern about stumbling influenced their walking. If these introspections are valid, confidence in the ability to walk safely without vision likely plays little role in the progressive overshooting.

We attempted to discriminate between adaptation effects and changes due to increased familiarity with the task by retesting the participants after a $10-\min$ period of visually guided walking. The results suggested that the participants remained comfortable with nonvisual walking. After eyesopen practice, blind-walking responses decreased for some targets, but not for others, leaving the relative contribution of adaptation versus familiarity unclear. Importantly, however, the progressive increases in walked distance in Experiment 2 were also associated with increased unintentional drifting in the marching-in-place task immediately after 37 blind-walking trials. In addition, we found virtually no correlation between signed walking errors and mean walking velocity, at any stage of the experiment. Taken together, we interpret these results as indicating that both drifting and progressive overshooting are largely the result of adaptation of self-motion perception brought about by the mismatch between locomotor activity and the lack of visual information signaling self-motion. The general ineffectiveness of eyes-open practice on reducing walked distances, however, may indicate that motor adaptation also plays a role in generating the progressive increases in walked distances. Interestingly, the amplitude of open-loop reaches to remembered targets also tends to increase over trials (Bingham, Zall, Robin, \& Shull, 2000; Vindras \& Viviani, 1998). This effect remains poorly understood in the domain of reaching, but perceptuomotor and/or motor adaptation are possible explanations.

What should be done to obtain an estimate of perceived location and/or distance that is minimally influenced by adaptation, changes in response strategy, and lack of familiarity? Ideally, participants should be exposed to nonvisual walking prior to data collection; on the basis of the point at which walking velocity leveled off in Experiment 2 (Trials $8-10$ ), approximately 3 min should suffice to adequately familiarize participants. Since this exposure could itself cause some adaptation to nonvisual walking, participants should then be allowed to walk with vision for a short time to prevent larger amounts of adaptation from accruing. Finally, the number of blind-walking trials after this eyes-open locomotion should be kept relatively low, to minimize additional recalibration and the possibility of changes in response strategy. Alternatively, breaks should be added periodically, with additional visually guided walking to minimize adaptation.

If participants undergo a nonvisual familiarization phase but are not reexposed to visually guided walking prior to testing, blind-walking responses are likely to overestimate participants' actual perceived location, because the responses are biased by adaptation processes; however, the response variability will likely be smaller than if no familiarization is given. If participants are not given a familiarization phase, the responses may initially underestimate 
participants' perceived location. However, over the course of many trials, adaptation (motor and/or perceptuomotor) will begin to lengthen the walked distances. To some extent, initial underestimates of perceived location due to lack of familiarity with the task will tend to be offset by later increases due to adaptation. Thus, averaging across trials may provide a reasonable compromise, although this method comes at the expense of increased response variability.

\section{AUTHOR NOTE}

This work was supported in part by NIH Grant RO1 NS052137 to J.W.P. The authors thank Deanne Adams, Margaret Cerullo, Thom Gennaro, Jessica Huang, Richard Lewis, Zita Patai, Shayna Thomas, and Petra Zdenkova for their assistance in data collection. Correspondence concerning this article should be addressed to J. W. Philbeck, Department of Psychology, George Washington University, 2125 G Street, N.W., Washington, DC 20052 (e-mail: philbeck@gwu.edu).

\section{REFERENCES}

Allen, G. L., Kirasic, K. C., Rashotte, M. A., \& Haun, D. B. M. (2004). Aging and path integration skill: Kinesthetic and vestibular contributions to wayfinding. Perception \& Psychophysics, 66, 170-179.

ANDRe, J., \& Rogers, S. (2006). Using verbal and blind-walking distance estimates to investigate the two visual systems hypothesis. Perception \& Psychophysics, 68, 353-361.

Anstis, S. (1995). Aftereffects from jogging. Experimental Brain Research, 103, 476-478.

BARLOW, H. B. (1990). A theory about the functional role and synaptic mechanism of visual after-effects. In C. Blakemore (Ed.), Vision: Coding and efficiency (pp. 363-375). New York: Cambridge University Press.

Bigel, M. G., \& Ellard, C. G. (2005). The contribution of nonvisual information to simple place navigation and distance estimation: An examination of path integration. Canadian Journal of Experimental Psychology, 54, 172-185.

Bingham, G. P., Zall, F., Robin, D., \& Shull, J. A. (2000). Distortions in definite distance and shape perception as measured by reaching without and with haptic feedback. Journal of Experimental Psychology: Human Perception \& Performance, 26, 1436-1460.

Creem-Regehr, S. H., Willemsen, P., Gooch, A. A., \& Thompson, W. B. (2005). The influence of restricted viewing conditions on egocentric distance perception: Implications for real and virtual indoor environments. Perception, 34, 191-204.

Durgin, F. H., \& Pelah, A. (1999). Visuomotor adaptation without vision? Experimental Brain Research, 27, 12-18.

Durgin, F. H., Pelah, A., \& Amiruddin, S. (1998). Measures of visuomotor adaptation to anomalous optic flow [Abstract]. Investigative Ophthalmology \& Visual Science, 39, S1094.

Durgin, F. H., Pelah, A., Fox, L. F., Lewis, J., Kane, R., \& WalLEY, K. A. (2005). Self-motion perception during locomotor recalibration: More than meets the eye. Journal of Experimental Psychology: Human Perception \& Performance, 31, 398-419.

Ellard, C. G., \& Shaughnessy, S. C. (2003). A comparison of visual and nonvisual sensory inputs to walked distance in a blind-walking task. Perception, 32, 567-578.

ELLIOTT, D. (1987). The influence of walking speed and prior practice on locomotor distance estimation. Journal of Motor Behavior, 19, 476-485.

Gilinsky, A. (1951). Perceived size and distance in visual space. Psychological Review, 58, 460-482.

Loomis, J. M., Da Silva, J. A., Philbeck, J. W., \& Fukusima, S. S. (1996). Visual perception of location and distance. Current Directions in Psychological Science, 5, 72-77.

Loomis, J. M., \& Philbeck, J. W. (2008). Measuring perception with spatial updating and action. In R. L. Klatzky, M. Behrmann, \&
B. MacWhinney (Eds.), Embodiment, ego-space, and action (pp. 1-43). Mahwah, NJ: Erlbaum.

Melvill Jones, G., Fletcher, W. A., Weber, K. D., \& Block, E. W. (2005). Vestibular-podokinetic interaction without vestibular perception. Experimental Brain Research, 167, 649-653.

Mittelstaedt, M.-L., \& Mittelstaedt, H. (2001). Idiothetic navigation in humans: Estimation of path length. Experimental Brain Research, 139, 318-332.

Mohler, B., Thompson, W. B., Creem-Regehr, S. H., Willemsen, P., PICK, H. L., JR., \& Rieser, J. J. (2007). Calibration of locomotion due to visual motion in a treadmill-based virtual environment. $A C M$ Transactions on Applied Perception, 4, 20-32.

Philbeck, J. W., \& Loomis, J. M. (1997). Comparison of two indicators of perceived egocentric distance under full-cue and reduced-cue conditions. Journal of Experimental Psychology: Human Perception \& Performance, 23, 72-85.

Philbeck, J. W., Loomis, J. M., \& Beall, A. C. (1997). Visually perceived location is an invariant in the control of action. Perception \& Psychophysics, 59, 601-612.

Pick, H. L., Jr., Rieser, J. J., Wagner, D., \& Garing, A. E. (1999). The recalibration of rotational locomotion. Journal of Experimental Psychology: Human Perception \& Performance, 25, 1179-1188.

Proffitt, D. R., Stefanucci, J., Banton, T., \& Epstein, W. (2003). The role of effort in perceiving distance. Psychological Science, 14, 106-112.

Rieser, J. J., Ashmead, D. H., Talor, C. R., \& Youngquist, G. A. (1990). Visual perception and the guidance of locomotion without vision to previously seen targets. Perception, 19, 675-689.

Rieser, J. J., Pick, H. L., JR., Ashmead, D. H., \& Garing, A. E. (1995). Calibration of human locomotion and models of perceptual-motor organization. Journal of Experimental Psychology: Human Perception \& Performance, 21, 480-497.

SCHWARTZ, M. (1999). Haptic perception of the distance walked while blindfolded. Journal of Experimental Psychology: Human Perception \& Performance, 25, 852-865.

Steenhuis, R. E., \& Goodale, M. A. (1988). The effects of time and distance on accuracy of target-directed locomotion: Does an accurate short-term memory for spatial location exist? Journal of Motor Behavior, 20, 399-415.

Sun, H.-J., Campos, J. L., Young, M., Chan, G. S. W., \& Ellard, C. G. (2004). The contributions of static visual cues, nonvisual cues, and optic flow in distance estimation. Perception, 33, 49-65.

Thomson, J. A. (1980). How do we use visual information to control locomotion? Trends in Neurosciences, 3, 247-250.

VindRas, P., \& ViVIani, P. (1998). Frames of reference and control parameters in visuomanual pointing. Journal of Experimental Psychology: Human Perception \& Performance, 24, 569-591.

Wu, B., OoI, T. L., \& HE, Z. J. (2004). Perceiving distance accurately by a directional process of integrating ground information. Nature, 428, 73-77.

\section{NOTES}

1. Data from 2 other participants were not included in the analyses. One individual appeared to not fully understand the task, producing highly variable responses that sometimes greatly exceeded the target distance (e.g., walking $39 \mathrm{~m}$ for the $16-\mathrm{m}$ target). Another individual produced responses that were more consistent but were significantly larger than those of the other participants. This participant's mean signed error, expressed as a percentage of the target distance, was $31.8 \%$, which was $2.67 S D$ units above the mean of $2.2 \%$ for the other participants.

2. One participant took two giant steps for the first presentation of the target at $4 \mathrm{~m}$, rather than walking at a normal pace as instructed. This data point was removed, so no $S D$ could be calculated.

3. The number of paces for 1 participant was not recorded in a trial following eyes-open practice.

(Manuscript received June 29, 2007; revision accepted for publication May 29, 2008.) 